\title{
Rilievo 3D e modellazione avanzata nello studio dei Forti di Roma: il Forte Monte Antenne
}

3D survey and advanced modeling in the study of the Forts of Rome: the Forte Monte Antenne

\section{Giovanna Spadafora a , Gabriele Bellingeri ${ }^{\text {b }}$, Marco Canciani ${ }^{\text {c }}$ Elisabetta Pallottino ${ }^{\text {d }}$, Simone Ferretti ${ }^{\text {e }}$, Eleonora Antonucci ${ }^{\mathrm{f}}$, Roberto Dolfini ${ }^{\mathrm{g}}$}

${ }^{a}$ Dipartimento di Architettura Università degli Studi Roma Tre, Rome, Italy, giovanna.spadafora@uniroma3.it

b Dipartimento di Architettura Università degli Studi Roma Tre, Rome, Italy, gabriele.bellingeri@uniroma3.it

${ }^{\mathrm{c}}$ Dipartimento di Architettura Università degli Studi Roma Tre, Rome, Italy, marco.canciani@uniroma3.it

${ }^{\mathrm{d}}$ Dipartimento di Architettura Università degli Studi Roma Tre, Rome, Italy, elisabetta.pallottino@uniroma3.it

e,f,g Associazione di Promozione Sociale Progetto Forti, Rome, Italy, info@ progettoforti.it

\begin{abstract}
In the studies the authors are conducting on the entrenched camp of Rome, 3D surveys and digital models are used as means to understand constructions with the aim of developing restoration and reutilization projects. For Forte Monte Antenne (1882-1891), the authors have carried out systematic studies of the formal and structural aspects. The data acquired from a direct survey and with laser scanner, drone and photogrammetry, integrated with data obtained from iconographic and bibliographic sources, were integrated in the creation of a digital model, which made the classification of the various elements in a structured database possible, including the verification of the relationship among the parts at varying levels, and the system of aeration ducts. Convective motions and the thermo-hygrometric and visual comfort within some of the environments, as well as thermographic surveys of the walls were conducted. The BIM model was integrated, therefore, with a MEP model. The hypothesis of restoration and possible adaptation to new functions cannot disregard the analysis of those parameters which complete the picture of environmental quality and thus of the effective potentials in repurposing of the structure.
\end{abstract}

Keywords: Forte Monte Antenne, 3D survey, environmental quality, modeling.

\section{Introduzione}

Nell'ambito degli studi che gli autori stanno conducendo, volti alla redazione di un Manuale del recupero dei Forti di Roma, il rilievo 3D e la modellazione digitale sono impiegati come strumenti di conoscenza dei manufatti, finalizzati alla redazione di progetti di restauro e riuso. La metodologia di analisi messa a punto intende infatti sottolineare le peculiarità di ciascun Forte, con una possibile sinergia rispetto agli intenti più volte manifestati dalle Amministrazioni lo- cali e dagli Enti dello Stato di riconnetterne gli spazi al disegno e alla vita della città. I Forti e i relativi compendi, oramai inglobati all'interno della città consolidata, restano estranei e invisibili nonostante le dimensioni imponenti (alcuni di essi occupano una superficie confrontabile a quella dell'invaso del colonnato della basilica di San Pietro). Attualmente, dei 15 Forti del campo trincerato, il Forte Monte Antenne è l'unico di proprietà di Roma Capitale, ora in gestione al 
Municipio II, 5 sono in consegna a Roma Capitale (Boccea, Portuense, Bravetta, Ardeatina e Prenestina), mentre gli altri -unitamente alle tre batterie Appia Pignatelli, Porta Furba e Batteria Nomentana- sono in consegna ai diversi corpi armati dello Stato. Realizzati in un arco di tempo piuttosto breve (tra il 1877 e il 1891), i Forti presentano caratteri simili -sono variazioni del forte prussiano tipo, adattato ai contesti morfologici e alle necessità belliche contingenti- e sono accomunati dall'adozione di una medesima tipologia muraria (con differenze dei paramenti esterni) e dall'utilizzo di pochi elementi con funzione decorativa, che presentano alcune varianti tra un Forte e l'altro. Risulta pertanto evidente come, anche in funzione di uno studio comparato tra $\mathrm{i}$ diversi manufatti, la costruzione di un modello BIM, parametrico, si configuri come lo strumento principe di indagine e di analisi, dinamico e implementabile. Esso è stato costruito sul caso specifico del Forte Antenne, nella consapevolezza che ogni studio di questo tipo non può che partire dalle singole specificità, e che ogni generalizzazione, se mal si applica alla modellazione e simulazione "del nuovo", ancor meno si adatta a quella delle costruzioni storiche -HBIM (heritage BIM)- che è caratterizzata dalla interdipendenza tra l'ambiente digitale a oggetti e i dati di acquisizione con metodo laser o fotogrammetrico (Donato, et al., 2017). Per la costruzione del modello BIM del Forte Monte Antenne, si sono rivelati essenziali lo studio della manualistica sulle fortificazioni e la lettura delle componenti architettoniche condotta attraverso i disegni di archivio conservati presso l'ISCAG (Istituto Storico e di Cultura dell'Arma del Genio). Il confronto fra teoria e progetto ha orientato le fasi di conoscenza dell'opera, guidando le indagini all'interno e all'esterno del manufatto militare. Il rilievo 3D, realizzato mediante laser scanner, è stato integrato con i dati desunti dal rilevamento diretto, confluiti, preliminarmente, in disegni bidimensionali. Murature, portoni di ingresso ai ricoveri dei soldati, cornici e serramenti in ferro, sono stati disegnati fino alla scala $1: 1$, avendo cura che la rappresentazione grafica fosse efficace nella annotazione della conoscenza acquisita ai fini del restauro, e quindi utile alla successiva modellazione degli oggetti tridimensionali (Fig.
1). Le immagini restituite dal volo del drone hanno consentito di ricucire gli spazi esterni, oggi accessibili, con le terre dei rampari e con il perimetro del fossato, attualmente infestati da alberi e arbusti. Per l'implementazione del modello, sono stati condotti studi sistematici sugli aspetti formali e strutturali e su quelli inerenti i sistemi passivi originari di ventilazione naturale e di smaltimento delle acque meteoriche, allo scopo di verificare la possibilità di una loro riattivazione (Spadafora, et al., 2018). Contestualmente sono stati individuati, attraverso il rilievo, quegli elementi, comuni a tutti i Forti, che concorrono alla costruzione di una libreria di oggetti parametrici.

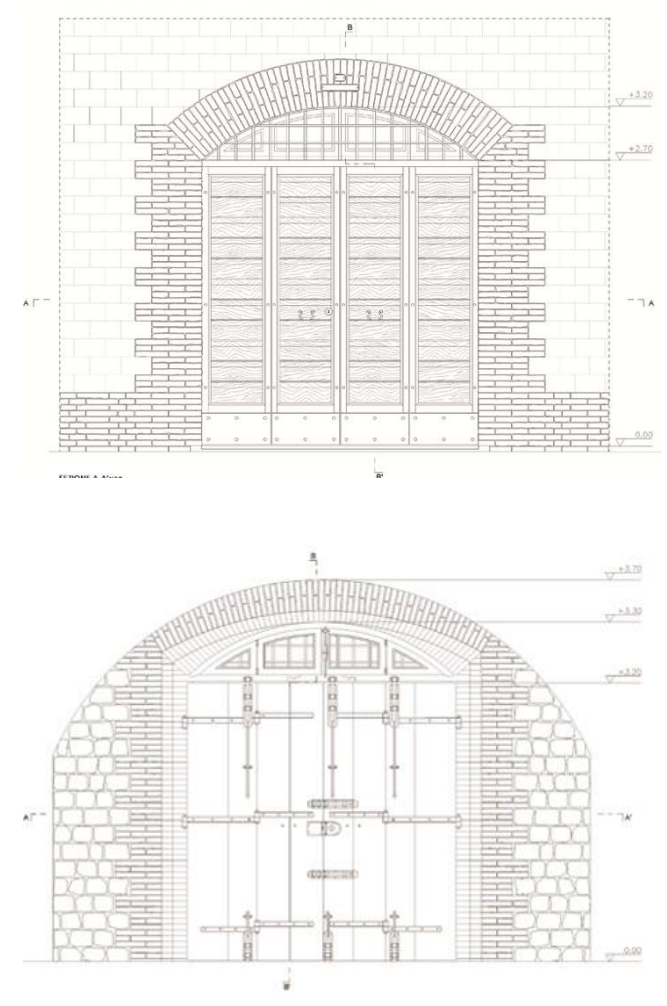

Fig. 1. Portone alla mercantile (prospetto esterno e interno). Ipotesi di ripristino.

Riguardo a questo tema, infatti, la creazione di un HBIM per i Forti del campo trincerato di Roma rende concreta l'aspirazione alla creazione di librerie parametriche appositamente create per gli edifici storici, perché in questo caso è riferita 
a edifici costruiti in un arco di tempo ristretto e contempla un numero "finito" di elementi e di loro possibili variazioni, da costruire in relazione alla manualistica e alle tecniche costruttive dell'epoca. Per ciascun elemento notevole del Forte è stato creato un database non secondo una acritica tassonomia, ma selezionando ciò che è stato ritenuto utile a una maggiore comprensione del manufatto o del singolo elemento: fotografie dello stato attuale, fotografie storiche, disegni tratti dalla manualistica, descrizione del rilevamento effettuato, disegni bidimensionali dettagliati desunti dal rilevamento, modelli tridimensionali, ulteriori dati come, ad esempio, risultati delle analisi diagnostiche, anche strumentali, analisi degli elementi di dissesto e di degrado.

\section{Il Modello BIM del Forte Monte Antenne}

La conformazione volumetrica dei Forti è articolata e complessa. Nel caso di studio, stante anche il cattivo stato di conservazione di parte delle strutture, delle terre e del fossato, la costruzione di un modello tridimensionale ha avuto come obiettivo (Bim use) primario la comprensione dell'organismo architettonico, sia nelle relazioni strutturali tra gli ambienti posti a quote diverse, sia nel funzionamento dei sistemi di ventilazione e smaltimento delle acque meteoriche.

La restituzione della consistenza del manufatto, attraverso la descrizione di tutti i suoi elementi costituenti, è stata condotta con lo scopo di analizzare il Forte come corpo organico, costituito di elementi su cui poter operare in fase di progetto di restauro (Brumana, et al., 2014). In relazione agli obiettivi della ricerca, si è proceduto quindi alla costruzione del modello digitale anteponendovi una riflessione critica, condensata in linee guida, ovvero in un BIM Execution Plan (identificato nelle normative nazionali come pGI, piano per la Gestione Informativa), nelle quali, tra l'altro, è indicata la strategia di segmentazione delle parti utili alla descrizione del manufatto: murature, coperture voltate, solai e, a seguire, porte, finestre, ma anche caditoie per

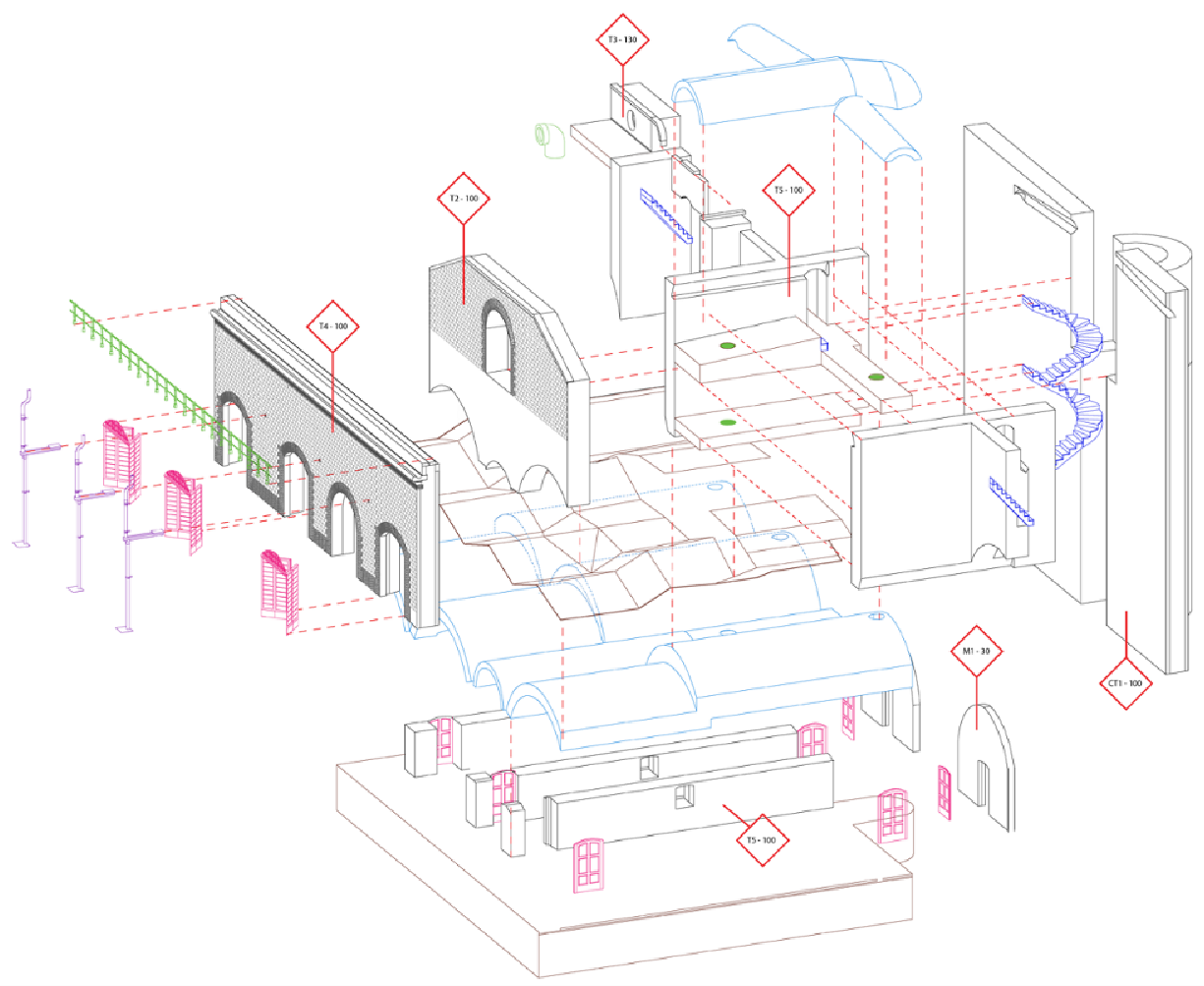

Fig. 2. Esploso assonometrico delle parti costituenti una porzione del fronte di testa del Forte Monte Antenne. 
l'acqua piovana, balaustre, griglie di ventilazione e via via elementi di maggior dettaglio (Fig. 2).

I sopralluoghi e il continuo confronto con la manualistica hanno consentito di arricchire il modello geometrico con specifiche informazioni sulla stratigrafia muraria, sulla composizione delle volte e dei solai, rendendoli così oggetti funzionali alla realizzazione di analisi performative. Esse sono state incluse nel pGI (piano di Gestione Informativa) come BIM Use secondari: analisi strutturali e analisi sullo sfasamento dell'onda termica, della trasmittanza e della ventilazione naturale; queste ultime, particolarmente interessanti nel caso dei Forti, dove il comportamento passivo è efficiente e rappresenta un tema di approfondimento significativo.

Una volta definite le componenti di base, è stato possibile realizzare un modello che, in quanto conforme alla effettiva morfologia del Forte, si configura come una simulazione costruttiva del manufatto (Fig. 3). Questo ha consentito di comprendere le relazioni tra le parti e gli allineamenti dei maschi murari lungo i diversi piani, nonché di rintracciare i canali di ventilazione e di ricostruirne il percorso all'interno delle murature e delle volte. Analogamente, grazie al modello, è stato ricostruito il sistema di smaltimento delle acque meteoriche (descritto in dettaglio in Spadafora, et al., 2018), fino alla definizione dell'aggancio della cornice di travertino alla muratura. Il modello è utile anche ai fini della classificazione e computazione degli elementi, a seguito della realizzazione di abachi dai quali dedurre quantità e dimensioni, anche ai fini di una loro eventuale riproducibilità in fase restauro.

Un modello siffatto consente, inoltre, di ottenere ulteriori livelli di approfondimento grazie all'associazione al modello architettonico di base di un MEP (Mechanical Electrical Plumbing),

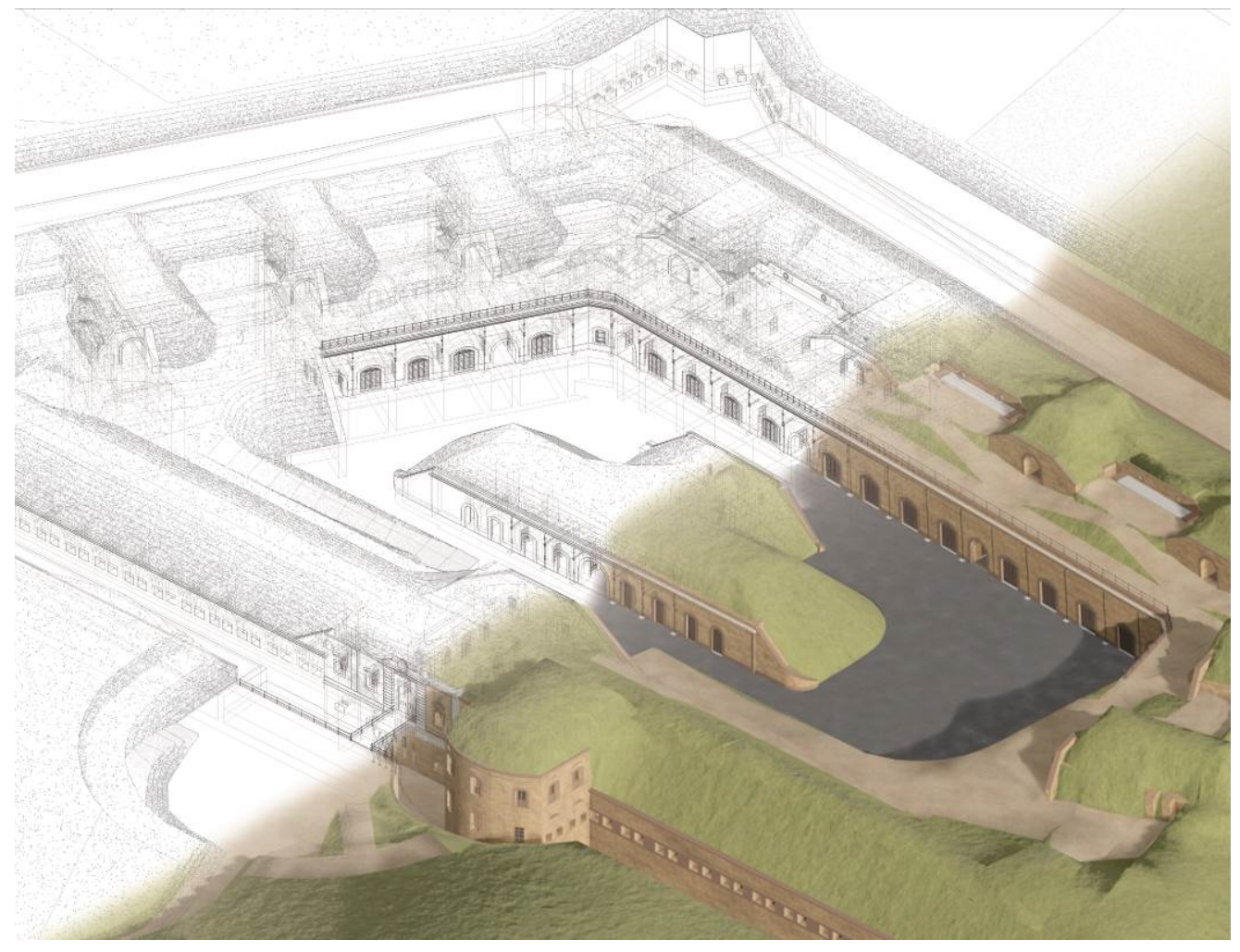

Fig. 3. Modello dello stato originario del Forte. 
che amplia le possibilità di analisi. Per il Forte Monte Antenne è stato realizzato un modello MEP semplificato (Fig. 4) che illustra il sistema di smaltimento dell'acqua piovana e una porzione del sistema di ventilazione passiva dei ricoveri di truppa; grazie a un modello di questo tipo è possibile non solo simulare il funzionamento dei sistemi a oggi, ma anche ipotizzarne possibili integrazioni migliorative e studiarne gli effetti (Fig. 5).

\section{La valutazione del comfort negli ambienti del Forte}

Nell'ipotesi che edifici storici possano essere riconvertiti oggi per destinazioni d'uso diverse da quelle per le quali sono stati concepiti, è opportuno potere valutare se e come sia possibile generare condizioni di comfort adeguate alle nuove funzioni. Spesso, raggiungere il comfort rivela estremamente oneroso in termini energetici, e quindi di costo, ma anche decisamente invasivo e poco adatto a mantenere il carattere originale degli ambienti interni.

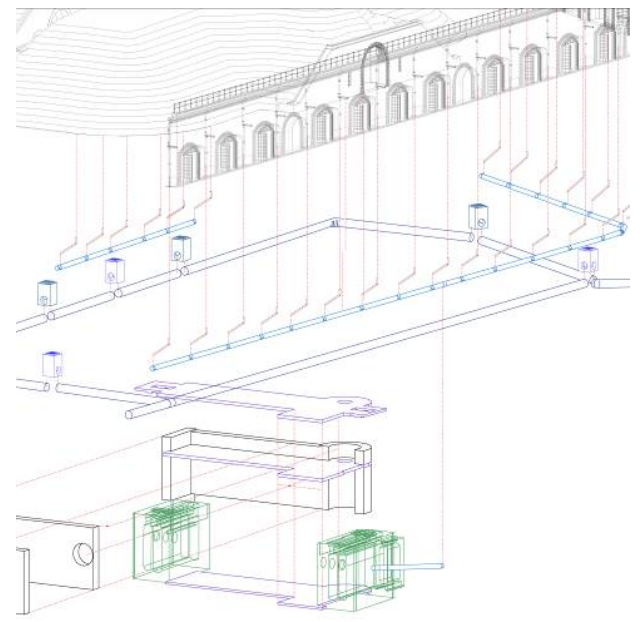

Fig. 4. Stralcio del modello MEP.

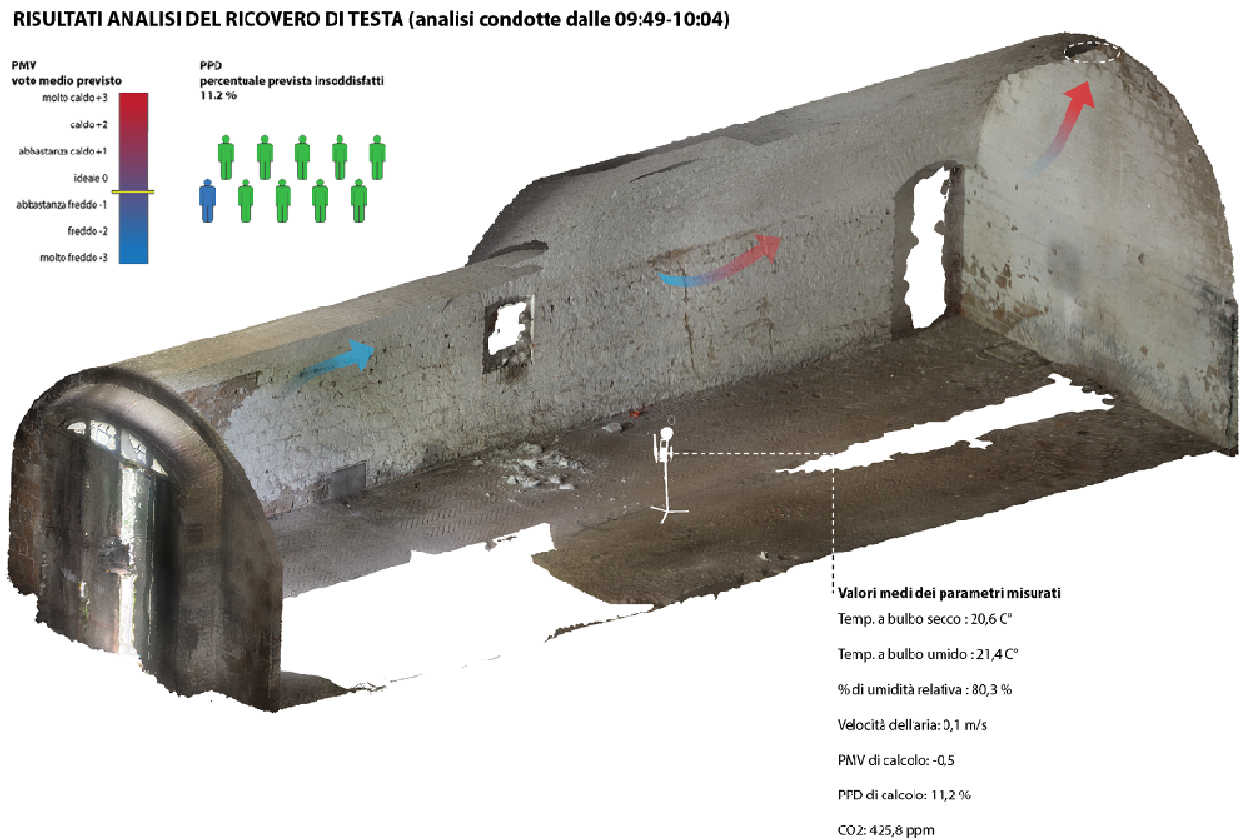

Fig. 5. Nuvola di punti di uno dei ricoveri di testa e analisi del comfort termo-igrometrico con conseguente valutazione del PMV. 
Risulta quindi di fondamentale importanza riuscire a conoscere l'edificio individuandone $\mathrm{i}$ problemi ma anche e soprattutto i potenziali punti di forza per la generazione del comfort in regime passivo o semi-passivo, per comprendere come e quanto possano essere in grado di garantire il comfort agli eventuali futuri utenti, così da poter ipotizzare un eventuale sistema impiantistico capace di operare in sinergia con l'edificio. Negli ambienti del Forte Monte Antenne sono stati realizzati anche rilievi strumentali volti a comprenderne il comportamento dal punto di vista fisico tecnico. In particolare sono state realizzate: rilevazioni termografiche al fine di rilevare eventuali patologie legate alle infiltrazioni di acque meteoriche; rilevazioni di temperatura umidità e velocità dell'aria in diversi periodi dell'anno $\mathrm{e}$ in diversi ambienti (Fig. 6) dell'edificio al fine di comprendere il rapporto tra le condizioni climatiche esterne e i parametri rilevati all'interno; misurazioni della velocità dell'aria relativa ai moti convettivi che si instaurano per convezione naturale, con particolare attenzione ai condotti verticali che fungono da camini di ventilazione, al fine di valutare le portate di aria di ricambio naturale. L'acquisizione di questi dati è pensata per consentire la taratura e la validazione di modelli di simulazione del comportamento dell'edificio. Il lavoro avviato in questo caso di studio rappresenta un primo tassello per la messa a punto di una metodologia sperimentale per la valutazione della potenziale idoneità di un Forte ad accogliere nuove funzioni, garantendo le necessarie condizioni di comfort agli utenti.

\section{Il ponte levatoio del Forte: un esempio di modello digitale a oggetti parametrici}

La complessità del processo di conoscenza di un organismo architettonico, nonché della sua rappresentazione secondo il grado di comprensione raggiunto, è commisurata alla peculiarità ed eterogeneità della consistenza del costruito, espressa da componenti sia materiali che immateriali le quali, nel caso del Forte Monte Antenne e in generale nell'edilizia storica, si articolano insieme alle trasformazioni intercorse tra uso e disuso dei suoi spazi e alle alterazioni dovute ai fenomeni di degrado occorsi nel tempo. Tra tutte le com- ponenti architettoniche del Forte, il ponte levatoio risulta l'elemento ossidionale che più si presta a essere rappresentato attraverso la modellazione a oggetti, che ne restituisce anche la meccanica.
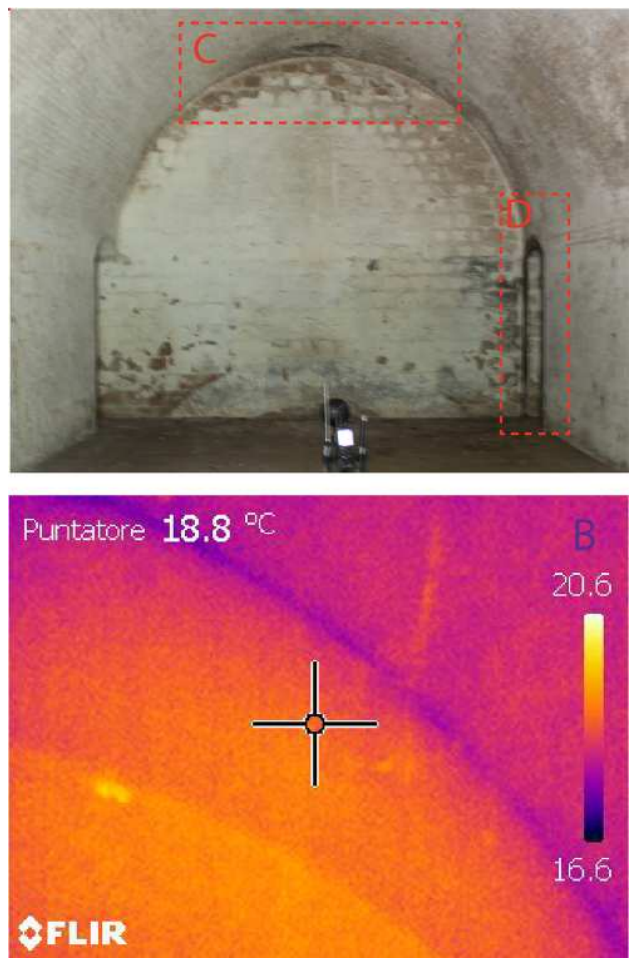

Fig. 6. Termografie scattate all'interno di uno dei ricoveri di testa.

Possiamo ricondurre la tipologia del ponte allo schema "a leva inferiore o a bilico" secondo un progetto, generalmente applicato a tutto il campo trincerato di Roma, che ha adottato nei suoi materiali ed elementi costruttivi innovazioni tecniche tipiche dell'epoca, come la standardizzazione e la prefabbricazione industriale delle componenti in ferro e ghisa (Scarambone, 1839). I ponti dei Forti romani differiscono in funzione di due parametri, la luce del ponte, determinata dalla larghezza del fossato, e l'altezza delle colonne di sostegno della parte fissa, dimensionata in base alla profondità del piano di fondazione del plinto (Spadafora, et al., 2018). 


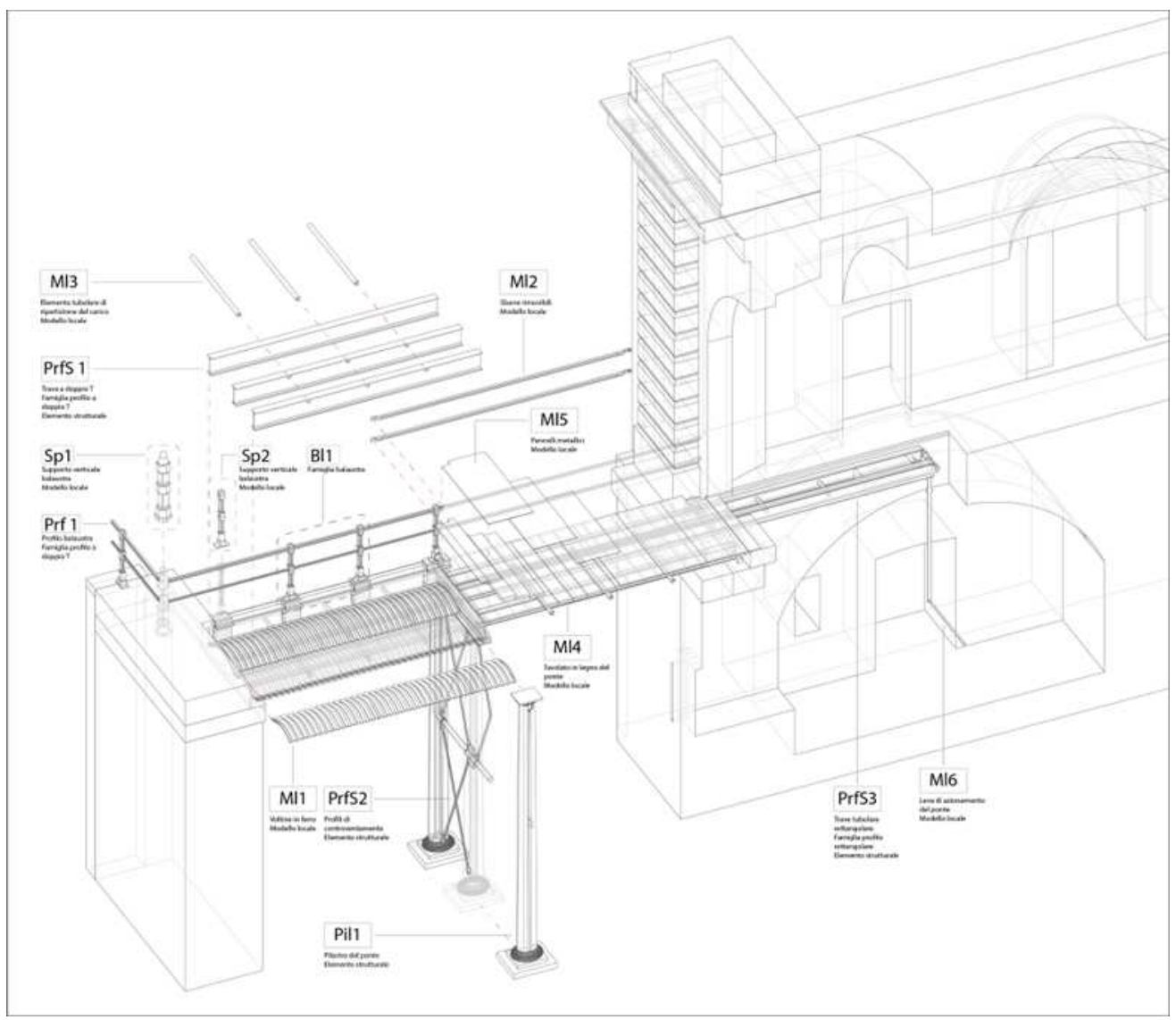

Fig. 7. LOD 400 del ponte levatoio.

Proprio queste caratteristiche hanno condotto alla modellazione parametrica di alcuni elementi (Fig. 7) (Fai, Rafeiro, 2014). Per il rilevamento diretto dell'intera struttura si è proceduto con la fotogrammetria integrata dal rilevamento diretto. Lo stato di conservazione generale ha permesso di identificare tutti gli elementi strutturali primari in ferro e ghisa, presenti nella loro conformazione e funzione originaria seppur piuttosto ammalorati, e di risalire agli elementi secondari mancanti o rimpiazzati da oggetti incongruenti. Gli oggetti tridimensionali, connotati e organizzati in gruppi, corrispondono puntualmente ai materiali che potrebbero essere quantificati e poi restaurati o sostituiti in vista di un fattibile ripristino del movimento del ponte levatoio. A questo proposito, l'analisi dello stato di degrado ha rivelato un diffuso ammaloramento degli elementi metallici dovuto all'umidità del fossato che ha provocato l'ossidazione prolungata del ferro e la conseguente formazione di ruggine che si manifesta in forma friabile sulle ali delle travi inferiori (mai illuminate dalla luce del sole, dove il processo di corrosione è ancora attivo) e in forma compatta sugli elementi superiori esposti alla luce del sole e soggetti a un'umidità minore. In alcuni punti, come sul dorso di varie voltine centinate, il ferro è completamente sgretolato, e può compromettere la resistenza meccanica degli elementi strutturali. Altre tipologie di degrado sono dovute a incrostazioni biologiche che agiscono sulle pellicole di protezione superficiali dei metalli, producendo sostanze corrosive e depositi solidi. 


\section{Conclusioni}

Il modello descritto è parte integrante del lavoro di redazione, in corso, del Manuale di recupero dei Forti di Roma (attività condotta in collaborazione con la Soprintendenza Archeologia Belle Arti e Paesaggio del Comune di Roma e l'Associazione di Promozione Sociale Progetto Forti) perché contribuisce a una più chiara definizione dei loro elementi costituenti. Una base informativa, interdisciplinare, che intende restituire l'accurato lavoro di indagine conoscitiva e porsi come strumento utile a coadiuvare le decisioni in merito al destino futuro di un patrimonio di grande rilevanza storico-architettonica.

\section{Note}

Tale attività di ricerca ha condotto alla firma di un accordo di collaborazione scientifica con il
Municipio II, interessato ad avviare progetti di recupero e riutilizzo, seppure parziale, di alcuni ambienti del Forte.

I disegni sono stati elaborati in occasione di due tesi di laurea, discusse a luglio 2018: Eleonora Antonucci, Il Forte Monte Antenne a Roma. Il rilievo per il restauro, Relatrici Giovanna Spadafora, Elisabetta Pallottino, Correlatore Simone Ferretti.

Roberto Dolfini e Marco Miranda, Il forte Monte Antenne a Roma. Metodologie di indagine $e$ analisi di sistemi per un progetto di rifunzionalizzazione, Relatori Gabriele Bellingeri e Giovanna Spadafora, Correlatore Simone Ferretti.

Eleonora Antonucci è autrice delle immagini $1 \mathrm{e}$ 7. Le altre immagini sono di Roberto Dolfini.

\section{Bibliography}

Donato, V.; Biagini, C.; Bertini, G.; Marsugli, F. (2017). "Challenges and opportunities for the implementation of HBim with regards to historical infrastructures [...]", International Archives of the Photogrammetry, Remote Sensing and Spatial Information Sciences, vol. XLII-5/W1.

Fai, S.; Rafeiro, J. (2014). "Establishing an appropriate level of detail (LoD) for a building information model (BIM) - West Block, Parliament Hill, Ottawa, Canada", ISPRS Annals of the Photogrammetry, Remote Sensing and Spatial Information Sciences, II-5, pp. 123-130.

Oreni, D.; Brumana, R.; Della Torre, St.; Banfi, F.; Barazzetti, L.; Previtali, M. (2014). "Survey turned into HBIM: the restoration and the work involved concerning the Basilica di Collemaggio after the earthquake (L'Aquila)", ISPRS Annals of the Photogrammetry, Remote Sensing and Spatial Information Sciences, II-5, pp. 267-273.

Scarambone, L. (1839). Intorno a' ponti levatoi delle piazze di guerra: discorso di, Napoli dalla Reale tipografia della guerra, Reale Tip. di Guerra, Napoli.

Spadafora G.; Ferretti S.; Canciani M.; Pallottino, E. (2018). "Il Forte Monte Antenne nel campo trincerato di Roma: studi e rilievi in corso", in Marotta, A.; Spallone, R., eds., FORTMED 2018. Defensive Architecture of the Mediterranean, Torino, vol. VIII. 